\title{
Summary of the Clinical Practice Guidelines for Diabetes Mellitus in Pregnancy in Myanmar
}

\author{
Tint Swe Latt, ${ }^{1}$ Than Than Aye, ${ }^{2}$ Moe Wint Aung, ${ }^{3} \mathrm{Ko} \mathrm{Ko}^{4}$ \\ ${ }^{1}$ University of Medicine 2, Yangon, Myanmar \\ 2Department of Medicine, University of Medicine 2, Yangon, Myanmar \\ ${ }^{3}$ Department of Medicine, University of Medicine 1, Yangon, Myanmar \\ ${ }^{4}$ Department of Endocrinology, University of Medicine 2, Yangon, Myanmar
}

\begin{abstract}
The aims of the Myanmar CPG for Diabetes in Pregnancy were to provide a framework to assist healthcare professionals in the management of diabetes in pregnancy and to aid primary care physicians in referrals to specialists, when necessary, in an appropriate manner. This article summarizes clinical practice guidelines developed for assisting Myanmar healthcare providers (physicians, endocrinologists, obstetricians, general practitioners) to better diagnose and effectively manage diabetes in pregnancy. It recommends a multidisciplinary approach in the management of diabetes in pregnancy in Myanmar.
\end{abstract}

Keywords: gestational diabetes mellitus, pregnancy, clinical practice guidelines, Myanmar

\section{INTRODUCTION}

Gestational diabetes accounts for $90 \%$ of diabetes in pregnancy. The prevalence of gestational diabetes mellitus in Myanmar by using WHO diagnostic criteria was $12.19 \%$, whereas the prevalence of GDM by using ADA criteria was $2.44 \%$ during the period of 1998 to $2003 .{ }^{1}$

In this context, healthcare providers from Myanmar should have relevant knowledge and skill for diagnosis and management of diabetes in pregnancy. This clinical practice guideline is developed for assisting Myanmar healthcare providers (physicians, endocrinologists, obstetricians, general practitioners) to better diagnose and effectively manage diabetes in pregnancy. It recommends a multidisciplinary approach in the management of diabetes in pregnancy in Myanmar.

\section{Summary of the Methodology for the Guideline Development}

A multidisciplinary working group consisting of endocrinologists, general physicians, primary care physicians, obstetrician and gynecologists, and other healthcare professionals from diverse disciplines developed the guideline.

The aims of the Myanmar CPG for Diabetes in Pregnancy were to provide a framework to assist healthcare professionals in the management of diabetes in pregnancy and to aid primary care physicians in referrals to specialists, when necessary, in an appropriate manner. The

\section{ISSN 0857-1074}

Printed in the Philippines

Copyright (c) 2013 by the JAFES

Received April 10, 2013. Accepted April 23, 2013. workgroup formulated these guidelines by reviewing published international guidelines and current evidence available in the literature, and considering the applicability of the CPG to the Myanmar population.

\section{Summary of Recommendations}

\section{Definition}

Gestational diabetes is any degree of glucose intolerance with onset or first recognition during pregnancy. It does not exclude the possibility that unrecognized glucose intolerance may have antedated or began concomitantly with the pregnancy.

Pre-gestational diabetes is pre-existing diabetes before pregnancy. 4

\section{Screening}

All pregnant mothers being seen at any OG or physician's clinic or by any healthcare provider should be evaluated for risk factors for type 2 diabetes and pre-diabetes.

\section{Recommendations:}

All pregnant women should be evaluated at the first prenatal visit for risk factors for diabetes. If patients already have FBS or RBS at the time of consultation, thresholds for DM will be the same as non-pregnant individuals. Screening for gestational diabetes using fasting plasma glucose, random blood glucose, glucose challenge test or urinalysis for glucose is not recommended. ${ }^{8}$

Corresponding author: Prof. Tint SweLatt, MBBS

President, Myanmar Society of Endocrinology and Metabolism

University of Medicine 2, Yangon, Myanmar

Tel. No.: 959-51673332

E-mail:proftsl@gmail.com 


\section{Gestational Diabetes Mellitus}

Screening Strategy to Detect GDM

Risk assessment should be done at first prenatal visit
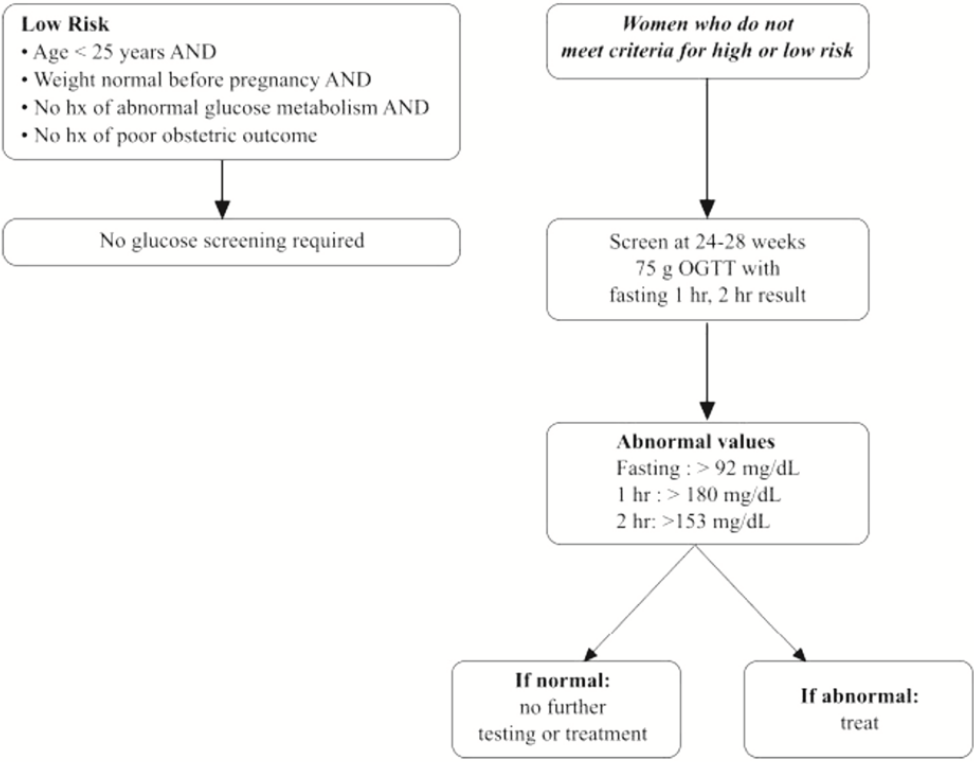

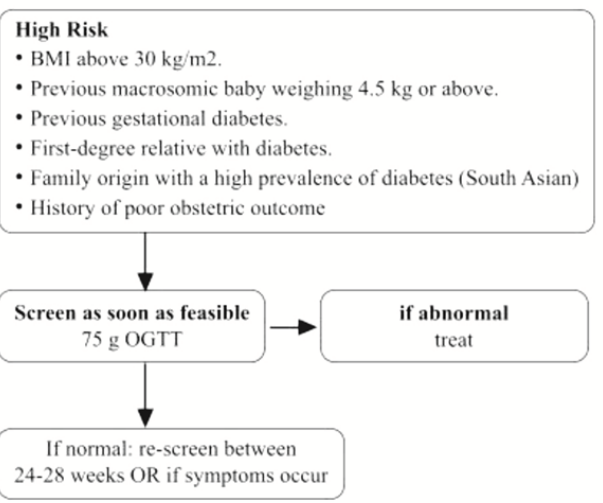

24-28 weeks OR if symptoms occur

Figure 1. Modified algorithm from Joslin Diabetes Center and Joslin Clinic Guideline for Detection and Management of Diabetes in Pregnancy ${ }^{7}$

Women with high risk for gestational diabetes should be screened by $75 \mathrm{~g}$ OGTT at initial visit.

If initial tests are negative for diabetes, OGTT should be repeated at 24-28 weeks of gestation. ${ }^{7}$

In women with low risk for gestational diabetes, screening is not advisable. ${ }^{7}$

Women who do not meet low or high-risk criteria should be screened at 24-28 weeks of gestation. ${ }^{7}$

Criteria for high risk are as follows: ${ }^{3}$

- BMI above $30 \mathrm{~kg} / \mathrm{m} 2$

- Previous macrosomic baby weighing $4.5 \mathrm{~kg}$ or above

- $\quad$ Previous gestational diabetes

- $\quad$ First-degree relative with diabetes

- Family origin with a high prevalence of diabetes (South Asian)

- Poor Obstetric outcome

Criteria for low risk are as follows: ${ }^{3}$

- Age $<25$ years AND

- Weight normal before pregnancy AND

- No history of abnormal glucose metabolism AND

- No history of poor obstetric outcome

\section{Diagnosis $^{8}$}

Recommendation: An oral glucose tolerance test (OGTT), preferably the $75 \mathrm{~g}$ OGTT, should be used to screen for gestational diabetes

\section{Interpreting the $75 \mathrm{~g}$ OGTT results \\ FBS $>92 \mathrm{mg} / \mathrm{dL}$ \\ 1-hour $\quad>180 \mathrm{mg} / \mathrm{dL}$ \\ 2-hour $\quad>153 \mathrm{mg} / \mathrm{dL}$}

*Any one value meeting threshold is considered gestational diabetes.

Women should have at least 10 hours fasting on the night prior to investigation.

\section{Targets for glycemic control and monitoring}

Women with gestational diabetes mellitus should be instructed to do self-monitoring of glucose.Target levels are much lower than normal people with diabetes.

Fasting sugar must be lower than 90 to $99 \mathrm{mg} / \mathrm{dL}$, 1-hour postprandial less than $140 \mathrm{mg} / \mathrm{dL}$ and 2 hour less than 120 $\mathrm{mg} / \mathrm{dL}{ }^{4}$

$\mathrm{HbA}_{1} \mathrm{C}$ should not be used routinely for assessing glycaemic control in the second and third trimesters of pregnancy. $^{3}$

\section{Management of diabetes in pregnancy}

\section{Recommendation}

Diabetes education including medical nutritional therapy is an essential component and recommended for all pregnancy with diabetes. They should be informed about the importance of good glycaemic control throughout 
pregnancy. It is also important to educate about insulin therapy and its injection technique. ${ }^{5,8}$

\section{Pre-conception management ${ }^{3,5}$}

Pre-conception care is critical for good outcomes for women with pre-gestational diabetes.

\section{Information and advice}

\section{Recommendation}

For all women with diabetes, information on the following should be offered in the pre-conception period:

- The importance of avoiding unplanned pregnancy should be an essential component of diabetes education

- Achieving and maintaining a healthy body weight.

- Diet and physical activity and the importance of an appropriate healthy eating meal plan

- The need for assessment and treatment of any complications of diabetes prior to conception and during pregnancy (retinal and renal assessment)

- The increased risk of congenital defects, neonatal morbidity and perinatal mortality associated with diabetes and pregnancy

- The risk of possible transient exacerbation of preexisting retinopathy or nephropathy.

- The risk of hypoglycaemia and of hypoglycaemia unawareness in pregnancy.

- The benefits of pre-conception glycaemic control at each contact

- Pregnancy-related nausea/vomiting and glycaemic control

- When to stop contraception

- Folic acid supplements (5 mg/day) from preconception until 12 weeks of gestation

- Review of, and possible changes to, medication, glycaemic targets and self-monitoring

- Diabetic women should have their glycaemic control $\left(\mathrm{HbA}_{1} \mathrm{C}\right)$ in optimal range before pregnancy.

\section{Glucose goals prior to conception ${ }^{2,3}$}

- Fasting and pre-meal plasma blood glucose: $80-110$ $\mathrm{mg} / \mathrm{dl}^{2}$

- 1 hour postprandial plasma blood glucose: 100-155 $\mathrm{mg} / \mathrm{dl}^{2}$

$$
\begin{aligned}
& \circ \quad \mathrm{A} 1 \mathrm{C}<7 \%^{2} \\
& \text { Avoid severe hypoglycemia }
\end{aligned}
$$

Blood pressure should also be well controlled, as there is also a high risk of preeclampsia and adverse fetal outcome.

Target blood pressure is $110-129$ systolic and 65-79 diastolic in women with chronic hypertension during pregnancy. Antihypertensives are initiated in pregnant patients with known or suspected chronic hypertension if blood pressure is $\geq 130 / 80$ three times during pregnancy. ${ }^{3}$
Stop ACEI and ARB. ${ }^{3}$

Stop statin.

- Antihypertensives that can be used during pregnancy are: ${ }^{3}$

o Alpha methyldopa

o Beta-blockers (acebutolol, sotalol; betaxolol, bisoprolol, labetalol, levatol, metoprolol,nadolol, timolol; atenolol - should not be used)

o Calcium channel blockers

It is important to check for microvascular and macrovascular complications throughout the pregnancy. Women with pre-existing diabetes should have ophthalmologic screening in the first trimester as worsening retinopathy can occur if sugar control is unsatisfactory. Those with underlying nephropathy should expect varying degrees of deterioration of renal function during pregnancy and the degree of proteinuria will also increase. Once the pregnancy is confirmed, women should attend a specialized joint antenatal diabetes clinic. $^{3}$

\section{Management during pregnancy ${ }^{8}$}

Most of the women with Gestational Diabetes can control blood glucose by diet and exercise only. Insulin is required in only $10-20 \%$ of cases not complying well with dietary management. The goal of dietary therapy is to avoid single large meals and foods with a large percentage of simple carbohydrates. The diet should include foods with complex carbohydrates and cellulose, such as unpolished rice, whole grain, bread, legumes and vegetables.

Patients with pre-existing diabetes should be switched from oral hypoglycemic agent(s) to insulin therapy at the onset of pregnancy. However, in those who don't have access to insulin therapy, they can continue metformin if sugar control is not beyond the target. Basal bolus insulin therapy is most preferred but twice daily premixed insulin regimen can be used alternatively if it matches the patient's normal glucose profile. Further, the regimen must be continuously modified as the pregnancy progresses from the first to the third trimester and insulin resistance rises. ${ }^{8}$

\section{Monitoring and screening fetal development ${ }^{3}$}

Offer:

- Ultrasound monitoring of fetal growth and amniotic fluid volume at 28 and 36 weeks

\section{Management during delivery ${ }^{3,8}$}

Those who have a normally grown fetus should be offered elective birth through induction of labour, or by elective caesarean section if indicated, after 38 completed weeks. 
During labour, capillary blood glucose should be monitored on an hourly basis in women with diabetes and should be maintained between 4 and $7 \mathrm{mmol} / \mathrm{litre}$ (72 to $126 \mathrm{mg} / \mathrm{dL}$ ). During intrapartum period, most will not need insulin glucose infusions in elective caesarean section, as the delivery time is not more than 30 minutes. Also patients whose diabetes are controlled well with diet also go through normal delivery without needing insulin infusion. It is crucial to monitor the neonate's sugar level in the first few hours after delivery to avoid hypoglycemia..$^{3,8}$

\section{Neonatal care}

The baby should stay with the mother unless extra neonatal care is required.

Do not transfer babies into community care until they are at least 24 hours old, maintaining their blood glucose levels and feeding well..$^{3,8}$

\section{Admission to a neonatal unit ${ }^{3}$}

Admit the baby to a neonatal unit if he or she:

- is hypoglycaemic with abnormal signs

- has respiratory distress or jaundice that requires monitoring or treatment

- has signs of cardiac decompensation, neonatal encephalopathy or polycythaemia

- needs intravenous fluids

- needs tube feeding (unless adequate support is available on the postnatal ward)

- $\quad$ is born before 34 weeks (or between 34 and 36 weeks if dictated clinically by initial assessment).

\section{Preventing, detecting and managing neonatal hypoglycaemia $^{3,8}$}

\section{Advise:}

- women to feed their babies as soon as possible (within 30 minutes of birth) and then at frequent intervals (23 hours) until pre-feeding blood glucose levels are maintained at $2 \mathrm{mmol} /$ litre or more.

- Test the baby's blood glucose levels 2-4 hours after birth

- if he or she has signs of hypoglycaemia, give intravenous dextrose as soon as possible

\section{Post natal care ${ }^{3}$}

After delivery most of the women with Gestational Diabetes will not need any hypoglycemic agents as insulin resistance improves. Women with insulin-treated preexisting diabetes should reduce their insulin immediately after birth and monitor their blood glucose levels carefully to establish the appropriate dose. At discharge, reinforce lifestyle management advice.

\section{Breastfeeding ${ }^{3}$}

Encourage breastfeeding (nutritional and immunological benefits to the baby). Advise women with type 1 diabetes or type 2 diabetes that self-monitoring should continue and good glycaemic control should be maintained during this period.

Insulin requirements drop immediately after delivery, and a dose adjustment will be needed to allow for the eating patterns of the breastfeeding mother.

Review medications, taking into consideration the potential risks associated with any transfer into the milk. Metformin and possibly glibenclamide (glyburide) may be used. Statins should be avoided, and consideration should be given to the choice of anti-hypertensive agents.

\section{Follow-up p $^{3,8}$}

Women who were diagnosed with gestational diabetes should be offered lifestyle advice (including weight control, diet and exercise) and offered a fasting plasma glucose measurement (but not an OGTT) at the 6-week postnatal check and annually thereafter. If the result is negative for diabetes, advise on high risk of future diabetes and on preventive lifestyle measures. Advise to check for diabetes every one to three years. If further pregnancies are planned, advise on the risk of developing GDM again, and the need for pre-pregnancy counseling.

\section{CONCLUSION}

This clinical practice guideline emphasizes the importance of multidisciplinary involvement in the management of diabetes mellitus in pregnancy and individualization of management according to the patient needs and available diagnosis and treatment options.

\section{References}

1. Tint SweLatt, 2003. A study of Gestational Diabetes Mellitus in Central Women Hospital in Yangon, a thesis for the Dr.Med.Sc (General Medicine), University of Medicine 1, Yangon.

2. American Diabetes Association. Diagnosis and classification of diabetes mellitus (Position Statement).Diabetes Care. 2013; 36 (Suppl 1): S11-S66.

3. National Collaborating Centre for Women's and Children's Health. NICE Clinical Guideline 63. Diabetes in pregnancy: Management of diabetes and its complications from pre-conception to the postnatal period. United Kingdom: National Institute for Health and Clinical Excellence, 2008.

4. IDF Clinical Guidelines Task Force. Global Guideline on Pregnancy and Diabetes. Brussels: International Diabetes Federation, 2009.

5. The HAPO Study Cooperative Research Group. Hyperglycemia and adverse pregnancy outcomes. N Engl J Med 2008; 358: 1991-2002.

6. Litonjua AD et al. ASEAN Federation of Endocrine Societies (AFES) Study Group on Diabetes in Pregnancy. PJIM 1996;34:37-42.

7. Joslin Diabetes Center and Joslin Clinic Guideline for Detection and Management of Diabetes in Pregnancy.Publications Department, 617-2265815. 2005

8. International Association of Diabetes and Pregnancy Study Groups Consensus Panel, Metzger BE, Gabbe SG, Persson B, Buchanan TA Catalano PA, Damm P, et al. International association of diabetes and pregnancy study groups recommendations on the diagnosis and classification of hyperglycemia in pregnancy. Diabetes Care.2010;33:67682.

9. Royal College of Obstetricians and Gynecologists; Diagnosis and Treatment of Gestational Diabetes.Scientific Impact Paper. No. 23, January 2011. 